\title{
Putra BINGO : from pen and paper to digital
}

\begin{abstract}
Gamification is one of the trends in education that is best suited to the current learning environment. Putra BINGO is a transformative assessment tool that uses gamification techniques. This research starts by elaborating the concept of Putra BINGO and its implementation using traditional method with pen and paper. The feedbacks gathered from the implementation are analyzed and applied to develop a multiplayer educational online game using Unity Game Engine. This paper describes details of the development for its two components; the database and the digital online game. Putra Bingo is available on PC and Android devices. It required additional plugins such as Photon Networking to handle game communication between multiple players. This is crucial to accommodate its test case application as an alternative assessment for teamwork skills using Rubrics and questionnaires. Further research will be conducted to enhance its functionality and user experience after the initial development of the digital online version is completed.
\end{abstract}

Keyword: Gamification; Games; Collaborative; Alternative assessment; Digital 